\title{
The Change in Parental Symptoms and Dysfunctional Cognitions in the Course of Trauma-Focused Cognitive-Behavioral Therapy: Sustainability Until One-Year Post-Treatment
}

\author{
Dunja Tutus, MSc, Elisa Pfeiffer, PhD, ${ }^{1}$ Paul L. Plener, MD, MHBA, ${ }^{1,2}$ Rita Rosner, $\mathrm{PhD},{ }^{3}$ \\ Dorothee Bernheim, $\mathrm{PhD}^{1}$, and Cedric Sachser, $\mathrm{PhD}^{1}$
}

\begin{abstract}
Objectives: Symptoms of distress and dysfunctional posttraumatic cognitions (PTCs) have been frequently described in parents of children and adolescents with posttraumatic stress symptoms (PTSS), especially if the parents had experienced traumatic events themselves. The inclusion of non-offending parents in trauma-focused cognitive-behavioral therapy (TFCBT) for children and adolescents may, thus, help parents to cope with the traumatic experience of their child. The aim of this study is to investigate the effects of TF-CBT on the parents, while taking their own history of traumatic experiences into account.

Methods: Parents ( $N=57,84.2 \%$ mothers) of children and adolescents who received TF-CBT completed the Posttraumatic Diagnostic Scale, the Beck Depression Inventory, the State-Trait Anxiety Inventory, and the Posttraumatic Cognitions Inventory. Treatment effects and the sustainability at 6- and 12 months post-treatment were tested via repeated-measures analysis of variance, following the intention-to-treat approach.

Results: $N=19(35.2 \%)$ of the parents evaluated their child's trauma as the worst event, 18 (33.3\%) rated their own experience as their worst event, and 17 (31.5\%) indicated that their own worst traumatic experience was the same type as their child's trauma. Significant improvements $(p<0.001)$ emerged for parental PTSS $[F(2,837)=8.27 ; d=0.30]$, depression $[F(3$, $284)=14.73 ; d=0.41]$, anxiety symptoms $[F(3,185)=17.44 ; d=0.64]$, and dysfunctional PTCs $[F(2,465)=13.58 ; d=0.46]$. Sustainability of these treatment gains remained at both follow-up time points $(p<0.05)$. There was no interaction between the time and the reference person of the traumatic index event, reported by parents.

Conclusion: These results indicate parental benefits from participation in TF-CBT delivered to their child, until 1-year post-treatment and independently from the parental trauma history. The ongoing tendency of improvement might indicate that TF-CBT furnishes children and their parents with skills to further reduce the impact of their traumatic memories. ClinicalTrials.gov NCT01516827.
\end{abstract}

Keywords: dysfunctional posttraumatic cognitions, parents, pediatric PTSD, sustainability of treatment gains, traumafocused cognitive-behavioral therapy

\section{Introduction}

$\mathbf{O}$ VERALL, $15.9 \%$ CHILDREN and adolescents (later referred to as children) exposed to any traumatic event develop posttraumatic stress disorder (PTSD; Alisic et al. 2014). It is also well known that a traumatic experience affects not only the child but also the family. Parents of traumatized children can, thus, be af- fected by the child's trauma exposure and may develop mental health problems themselves (Koplewicz et al. 2002), such as posttraumatic stress symptoms (PTSS), depression, anxiety, or dysfunctional posttraumatic cognitions (PTCs; Tutus and Goldbeck 2016).

A significant relationship between parental mental health problems and the child's PTSD is a robust finding (Morris et al. 2012)

\footnotetext{
${ }^{1}$ Department of Child and Adolescent Psychiatry/Psychotherapy, Ulm University, Ulm, Germany.

${ }^{2}$ Department of Child and Adolescent Psychiatry, Medical University of Vienna, Vienna, Austria.

${ }^{3}$ Department of Psychology, Catholic University of Eichstaett-Ingolstadt, Eichstätt, Germany.

This work is part of the "TreatChildTrauma" study, which received a grant from the German Federal Ministry of Education and Research (BMBF). Funding: Healthcare research (Funding Code: 01GY1141).
} 
and might be partially explained by a shared genetic vulnerability (Smoller 2016) and bidirectional effect between the symptoms in both, children and their parents (Koplewicz et al. 2002; Neill et al. 2018). There is evidence that parental symptoms remain stable until 30 months after the trauma exposure, even if the child's symptoms decrease (Laor et al. 1997). Nevertheless, parental mental health posttrauma might increase the risk for the development and maintenance of PTSD in the child (Trickey et al. 2012) and was found to mediate PTSS in preschool-age children (Scheeringa et al. 2015). However, the meta-analysis by Trickey et al. (2012) mostly included cross-sectional and retrospective studies instead of longitudinal studies, with control conditions that might derive more temporal relations of variables.

Especially dysfunctional PTCs can play a crucial role in the development and maintenance of trauma-related mental health problems in adults (Foa et al. 1999) as well as children (MeiserStedman et al. 2009). Recent research suggests that parental dysfunctional PTCs related to their child's trauma, such as: "The world is a dangerous place" are significantly positively associated with parental trauma-related mental health problems (Tutus and Goldbeck 2016).

In addition, parental dysfunctional PTCs may negatively model the child's appraisals of the traumatic event and thereby contribute to the maintenance of the child's PTSS (Hiller et al. 2018). Finally, parental PTCs are associated with the child's response to traumafocused treatment (Nixon et al. 2012). The change in dysfunctional PTCs was found to mediate the reduction in PTSS, in the course of the trauma-focused treatment for adults (Zalta et al. 2014), as well as in children (Pfeiffer et al. 2017; Jensen et al. 2018). Further, it has been shown that the change in parental PTCs related to their child's trauma, throughout the trauma-focused treatment delivered to the child, mediated parental perception of their child's PTSS post-treatment (Tutus et al. 2018). However, there was no mediation in child's PTSS, if assessed with clinical interview or using the child's self-report (Tutus et al. 2018).

Besides being affected by the child's traumatic experience, many parents report traumatic events themselves (Tutus and Goldbeck 2016). Elevated PTSS, depression, and anxiety symptoms have been observed in parents, whose children have experienced a traumatic event, which was either the same or similar to a traumatic event experienced by the parents (Scheeringa and Zeanah 2001). Thus, including parents in the child's trauma-focused treatment seems to be essential for successfully treating children affected by traumatic events.

Trauma-focused cognitive-behavioral therapy (TF-CBT), as manualized by Cohen et al. (2006a), is recommended as a first-line treatment for children suffering from PTSD (International Society for Traumatic Stress Studies [ISTSS], 2019) and seems to be beneficial even for the caregivers' own distress and symptoms precipitated by their child's trauma (Cohen et al. 2004b). TF-CBT is an evidence-based and well-established treatment (Morina et al. 2016; Dorsey et al. 2017), associated not only with significant reductions in PTSD, but also with a reduction of other trauma-related psychopathology such as depression and anxiety, behavioral problems, dysfunctional PTCs, and improvement of psychosocial functioning (Goldbeck et al. 2016; Morina et al. 2016) in children. Several studies worldwide have demonstrated the sustainability of treatment effects for children with PTSD, achieved by TF-CBT, over 1-year post-treatment (Cohen et al. 2005; Mannarino et al. 2012; Nixon et al. 2017; Jensen et al. 2017; Tutus et al. 2017b).

In contrast to other trauma-focused treatments, the inclusion of a caregiver plays a crucial role in this treatment. The inclusion of parents furnishes them with techniques and parenting skills that may help them not only to manage the problems of their children, but also to deal with their own symptoms and distress. In addition, parental participation allows them to optimally encourage their child in practicing these skills together, between treatment sessions (Cohen et al. 2006a).

There is a growing body of research on the effects of TF-CBT on participating parents, suggesting that treatment primarily focused on children may result in decreased symptom severity in the caregivers (Martin et al. 2019). In this context, TF-CBT was found to be associated with enhanced parenting efficacy (Deblinger et al. 1996, 2011; King et al. 2000; Cohen et al. 2004b), parent-child communication, and familial attachments (Cohen et al. 2006a).

TF-CBT was found to alleviate parental intrusive thoughts, negative emotional reactions (Deblinger et al. 2001), abuse-specific distress (Deblinger et al. 2011), PTSD (Deblinger et al. 2001; Cohen et al. 2004a, 2006b; Nixon et al. 2012), depression (Cohen et al. 2004a, 2004b, 2007; Deblinger et al. 2011; Nixon et al. 2012; Holt et al. 2014; Tutus et al. 2017a; Neill et al. 2018), and parental dysfunctional PTCs (Nixon et al. 2012; Tutus et al. 2018) after the child's trauma. Further, one meta-analysis showed that including parents in the children's treatments enhanced treatment effects for anxiety and depression, but it did not enhance the effects for PTSS or externalizing problems (Silverman and Hinshaw 2008).

A few studies have investigated parental symptoms at 6- and 12month follow-ups (MFU). In their dismantling study, Mannarino et al. (2012) evaluated four different TF-CBT treatment groups ( 8 vs. 16 sessions, with vs. without trauma narrative) in a sample of children who had experienced sexual abuse and their parents. They found that a reduction in parental depression was sustained until 12 MFU, whereas a change in parental emotional distress continued to improve during the follow-up periods, for the whole study sample and without any significant differences between the conditions. Similarly, Nixon et al. (2017) found, in their sample of children, who experienced a single-incident trauma and their parents, that reduction in parental depression, PTSD, and dysfunctional PTCs at post-treatment was sustainable at $12 \mathrm{MFU}$, without any significant difference between the condition with and without exposure. Deblinger et al. (2006) found that parents of sexually abused children who participated in TF-CBT continued to report less severe abusespecific distress, compared with parents whose children received child-centered therapy.

Further studies, conducted by independent researchers and within samples of various trauma types, are needed to extend the knowledge on the long-term effects of TF-CBT on participating parents. Assuming that parental own trauma history has an impact on their response to trauma exposure of their child, parental previous traumatic experiences needs to be taken into account while investigating parental benefit from TF-CBT and its long-term effects.

A recent systematic review on the role of caregiver psychopathology in TF-CBT recommends that future studies should report on caregiver demographic data, trauma history, and psychopathology, measured by psychometrically sound instruments and at multiple points in time, including mid-treatment (Martin et al. 2019).

Hence, the first aim of this study is to investigate the effects of TF-CBT on participating parents, regarding PTSS, depression, anxiety, and dysfunctional PTCs related to the trauma of the child, in the course of TF-CBT and until $12 \mathrm{MFU}$. Based on the literature described earlier, we expect a significant symptom reduction at post-treatment and sustainability of the treatment gains at $6 \mathrm{MFU}$ and $12 \mathrm{MFU}$. The second aim is to explore whether parental 
previous traumatic experiences have an impact on parental benefit from TF-CBT at both post-treatment and $6 \mathrm{MFU}$ and $12 \mathrm{MFU}$ regarding PTSS, depression, anxiety, and dysfunctional PTCs. Parents will, thus, be categorized into three groups based on their indicated index event: (1) parents, who rated one of their own experiences as their worst event; (2) parents, who evaluated their child's trauma as the worst event; and (3) parents, who indicated that their own worst traumatic event was the same type as their child's trauma.

\section{Methods}

\section{Participants and study design}

The current study sample consists of the non-offending birth parents $(N=57)$ of children randomized to the TF-CBT condition in a single-blind, parallel-group randomized controlled trial (RCT; Goldbeck et al. 2016). The sociodemographic characteristics of the participants are displayed in Table 1 . The children were recruited between February 2012 and January 2015 at three university and five community clinics for child and adolescent psychiatry and psychotherapy in Germany.

Table 1. Sample Description of Participating Parents ANd Their Children at Baseline

\begin{tabular}{|c|c|}
\hline Parents & $\mathrm{N}=57$ \\
\hline Gender: female $n(\%)$ & $48(84.2)$ \\
\hline Missing information $n(\%)$ & $1(1.8)$ \\
\hline Age in years $M(\mathrm{SD})$, min-max & 42.38 (7.07), 29-59 \\
\hline Missing information $n(\%)$ & $2(3.5)$ \\
\hline \multicolumn{2}{|l|}{ Educational level $n(\%)$} \\
\hline$<9$ Years school & $1(1.8)$ \\
\hline 9-11 Years school & $38(66.7)$ \\
\hline$\geq 12$ Years school & $17(29.8)$ \\
\hline Missing information & $1(1.8)$ \\
\hline Current employment $n(\%)$ & $40(70.2)$ \\
\hline Missing information $n(\%)$ & $1(1.8)$ \\
\hline \multicolumn{2}{|l|}{ Country of origin $n(\%)$} \\
\hline Germany & $46(80.7)$ \\
\hline Other countries & $10(17.5)$ \\
\hline Missing information & $1(1.8)$ \\
\hline \multicolumn{2}{|l|}{ Parents: $n(\%)$} \\
\hline Live together & $23(40.4)$ \\
\hline Do not live together & $33(57.9)$ \\
\hline Missing information & $1(1.8)$ \\
\hline $\begin{array}{l}\text { Number of experienced traumatic events } \\
M(\mathrm{SD}), \text { min-max }\end{array}$ & 2.04 (2.04), 0-7 \\
\hline \multicolumn{2}{|l|}{ Children and adolescents } \\
\hline Gender: female $n(\%)$ & $41(71.9)$ \\
\hline Age in years $M(\mathrm{SD}), \min -\max$ & $12.74(2.90), 6-17$ \\
\hline $\begin{array}{l}\text { Number of experienced traumatic } \\
\text { events, caregiver-report } M(\mathrm{SD}) \text {, } \\
\text { min-max }\end{array}$ & 1.47 (1.79), 0-11 \\
\hline \multicolumn{2}{|l|}{ CAPS-CA index event $n(\%)$} \\
\hline $\begin{array}{l}\text { Interpersonal trauma, accidental } \\
\text { trauma }\end{array}$ & $44(77.2), 13(22.8)$ \\
\hline CAPS-CA $M(\mathrm{SD}), \min -\max$ & 60.18 (18.02), 36-109 \\
\hline PTSD diagnosis, based on DSM-IV & $43(75.4)$ \\
\hline
\end{tabular}

PTSD, posttraumatic stress disorder; CAPS-CA, Clinician Administered PTSD Scale for Children and Adolescents, version for DSM-IV; DSM-IV, Diagnostic and Statistical Manual of Mental Disorders, 4th edition (American Psychiatric Association 1994); SD, standard deviation.
The children participating in the RCT showed clinically relevant PTSS and at least one symptom, each of re-experiencing, avoidance, and hypervigilance, assessed with the Clinician Administered PTSD Scale for Children and Adolescents (CAPS-CA; Nader et al. 2002), version for Diagnostic and Statistical Manual of Mental Disorders, 4th edition (DSM-IV; American Psychiatric Association 1994, at study admission. Children currently receiving another form of psychotherapy were not included in the RCT. During the RCT, all children were living in safe circumstances to minimize the risk of re-traumatization. All children and their parents were assessed at baseline, mid-treatment, and post-treatment, 6 MFU and 12 MFU. All children and their parents gave their informed written consent for participation in the study. The RCT received ethics approval from the Institutional Review Board at Ulm University and was registered under ClinicalTrials.gov.

\section{Intervention}

TF-CBT is a component-based manualized intervention, consisting of 12 weekly sessions, divided into three treatment phases: stabilization and skill building (Sessions 1-4), exposure to and cognitive processing of the trauma (Sessions 5-8), and fostering safety and future development (Sessions 9-12) (Cohen et al. 2006a). Each session, parallel or conjoint with patients and parents, took 90 minutes. In sessions with parental participation, the therapists spent about 45 minutes with the child and 45 minutes with the parent. All 26 study therapists read the treatment manual in German (Cohen et al. 2009), completed a certified web-based training program, and attended a 2-day personal training course in TF-CBT before starting delivering TF-CBT. During the RCT, they were supervised by the senior therapists at their study sites and by either one of the developers of the intervention or an approved TF-CBT trainer via biweekly conference calls (Goldbeck et al. 2016).

Treatment completers $(n=46 ; 80.7 \%)$ were defined as children who had completed at least eight therapy sessions, including psychoeducation, relaxation and affective modulation skills, cognitive restructuring and exposure, the common elements of the evidencebased trauma-focused interventions (Dorsey et al. 2011). In a subsample of the treatment completers, therapists spent about 45 minutes with the parents in $M=9.76$ (standard deviation=2.24) sessions. Altogether, $n=41(89.1 \%)$ children shared the trauma narrative in a conjoint session with at least one parent. The overall adherence to the manual, as measured via videos and session checklists, was confirmed in $96 \%$ of the evaluated sessions (Goldbeck et al. 2016).

\section{Instruments}

The Posttraumatic Diagnostic Scale (Foa 1995), German version (A. Ehlers, R. Steil, H. Winter, E.B. Foa EB, unpublished data) is a self-report measure of PTSD based on the DSM-IV (American Psychiatric Association 1994). For this study, we modified the instruction. Using the checklist with 12 potentially traumatic events, we asked the participating parents to indicate those events they and their child had experienced or witnessed. The parents who reported more than one event had to choose one event that was subjectively the worst for them (index event). When combining the parental and the child's lifetime trauma exposure, the worst event could be an event experienced only by the parent, only by the child or the same/similar traumatic event experienced by both. Afterward, the respondents were asked to rate how much they were affected by each of the symptoms when referring to the worst event. A response scale ranging from 0 (not at all or only one time) to 3 (five or more 
times a week/almost always) was applied. Internal consistency (Cronbach's $\alpha=0.91$ ) was excellent in our RCT sample (Tutus and Goldbeck 2016).

The Beck Depression Inventory-Second Edition (BDI-II; Beck et al. 1996), German version (Kühner et al. 2007), is a self-report measure of severity of depression based on the DSM-IV (American Psychiatric Association 1994). The BDI-II comprises 21 items with four response options, ranging from not present (0) to severe (3). Cronbach's $\alpha$ (0.92) was excellent in our sample (Tutus et al. 2017a).

The State-Trait Anxiety Inventory (STAI; Spielberger et al. 1970), German state version (STAI-G Form X 1; Laux et al. 1981) is a selfreport measure of anxiety. The STAI-G Form X 1 retains the 20 -item format with four response options for each item, ranging from 1 (not at all) to 4 (very much so). Cronbach's $\alpha$ for the whole scale (0.66) was acceptable in our sample (Tutus and Goldbeck 2016).

The Posttraumatic Cognitions Inventory (Foa et al. 1999) is a self-report measure that assesses dysfunctional PTCs and beliefs in the following domains: self, world, and self-blame. The items are rated on a Likert-type scale ranging from 1 (totally disagree) to 7 (totally agree). In this study, we used the original form of the questionnaire, translated into German, and instructed the parents to respond with regard to the worst event that their child had been exposed to. Cronbach's $\alpha$ for the whole scale (0.95) was excellent in our sample (Tutus and Goldbeck 2016).

\section{Data analyses}

Intention-to-treat analyses were performed to test our hypotheses. We used the expectation-maximization algorithm (Dempster et al. 1977) to impute missing dimensional data (PTSS, depression, anxiety, and dysfunctional PTCs). Little's Missing Completely At Random test indicated that these data were missing completely at random [PTSS: $\chi^{2}(30)=26.22, p=0.664$; depression: $\chi^{2}(22)=12.94, p=0.935$; anxiety: $\chi^{2}(21)=19.96, p=0.524$; PTCs: $\left.\chi^{2}(21)=9.02, p=0.989\right]$. To examine changes over the measurement time points (baseline, mid-treatment, post-treatment, $6 \mathrm{MFU}$ and $12 \mathrm{MFU}$ ), repeated-measures analysis of variances (ANOVAs) were performed for the dimensional outcomes, as dependent variables. Main effects were compared by using Bonferroni correction for multiple comparisons. We compared baseline scores with all other measurement time points. Cohen's $d$ was used as effect size estimation for pre-mid-treatment, pre-post-treatment, pre-6 MFU, and pre-12 MFU (Cohen 1988).

To explore an impact of parental previous traumatic experience on the treatment outcome, we formed three groups based on the index event reported by the parent: (1) parents who rated one of their own experiences as their worst event, (2) parents who evaluated their child's trauma as the worst event, and (3) parents who indicated that their own worst traumatic event was the same type as their child's trauma. This reference person (parent, child, or both) served as a group variable in the ANOVAs in a next step. The significance level for all statistical tests was set at $p<0.05$ (two-tailed). The statistical analyses were performed by using IBM SPSS Statistics 25 .

\section{Results}

Repeated-measures ANOVAs indicated statistically significant improvement for all outcome measures (Table 2). Pairwise comparisons revealed significant differences between baseline and post-treatment $(p=0.017), 6 \mathrm{MFU}(p=0.033)$, and $12 \mathrm{MFU}$ $(p<0.001)$ for PTSS. Effect sizes were small for pre-post comparisons and increased to medium effect sizes at $12 \mathrm{MFU}$ (Table 2).
Pairwise comparison, regarding depression, suggested significant differences between baseline and mid-treatment $(p=0.035)$, posttreatment ( $p=0.002), 6 \mathrm{MFU}(p<0.001)$, and $12 \mathrm{MFU}(p<0.001)$. Similar to PTSS, the small effect sizes tended to increase with every further measurement time point and reached medium effect size at 12 MFU. Pairwise comparison in regard to anxiety revealed significant differences between baseline and post-treatment, $6 \mathrm{MFU}$ and $12 \mathrm{MFU}$ $(p<0.001)$, with medium effect sizes at all three measurement time points. Similar results were found for dysfunctional PTCs while comparing baseline and post-treatment $(p=0.012), 6 \mathrm{MFU}$ ( $p=0.001)$, and $12 \mathrm{MFU}(p<0.001)$, with medium effect sizes at $6 \mathrm{MFU}$ and $12 \mathrm{MFU}$.

Taken together, parents' own history of traumatic experiences with their child's lifetime trauma exposure, 19 (35.2\%) parents evaluated their child's trauma as the worst event, $18(33.3 \%)$ rated their own experiences as their worst event, and 17 (31.5\%) indicated that their own worst traumatic event was the same type as their child's trauma. The remaining three parents did not report on any traumatic events and were excluded from the further analyses. There was no significant interaction between time and reference person of the parental traumatic index event for PTSS, anxiety, and dysfunctional PTCs, only for depression. However, main effect comparisons did not reveal any significant group difference regarding depression (Table 2).

\section{Discussion}

The purpose of this study was to investigate the long-term effects of TF-CBT regarding trauma-related symptoms in nonoffending parents who participated in the treatment delivered to their child. Our results confirm and extend previous findings regarding the parental benefit from participating in TF-CBT. In line with Mannarino et al. (2012) and Nixon et al. (2017), we found reductions in parents' depression at post-treatment and the sustainability of the treatment gains at $6 \mathrm{MFU}$ and $12 \mathrm{MFU}$. Consistent with Nixon et al. (2017), we found post-treatment reduction in parental PTSS and dysfunctional PTCs as well as sustainability of these findings at $12 \mathrm{MFU}$. This is in accordance with other RCTs on TF-CBT (Cohen et al. 2005; Mannarino et al. 2012; Nixon et al. 2017; Jensen et al. 2017; Tutus et al. 2017b), suggesting that longterm effects after receiving TF-CBT are not only typical for children, but also typical for their participating parents.

The ongoing tendency of improvement might indicate that TFCBT furnishes children and their parents with skills to reduce the impact of their traumatic experiences. Practicing skills during TFCBT and ongoing support by parents (Deblinger et al. 1999) may result in greater self-confidence (Mannarino et al. 2012) and further consolidation of mental health after treatment. The reduction of dysfunctional PTCs might be responsible for the long-term effects of TF-CBT (Foa et al. 1999; Zalta et al. 2014). Scher et al. (2017) reported from their study with adults that dysfunctional PTCs are associated with symptoms up to 10 years after cognitive behavioral treatment for PTSD and proposed their reduction as a potential mechanism for long-term maintenance of treatment gains. Another explanation for parental improvement might be a bidirectional effect between parents and their children, which might result in similar treatment effects in both, or even the possibility that the child's symptom reduction positively impacted their parentś mental health, rather than vice versa, as speculated by Koplewicz et al. (2002).

Regarding the impact of the parental trauma history on their improvement, we could not find any group difference, based on the reference person of the index event, regarding parental PTSS, 


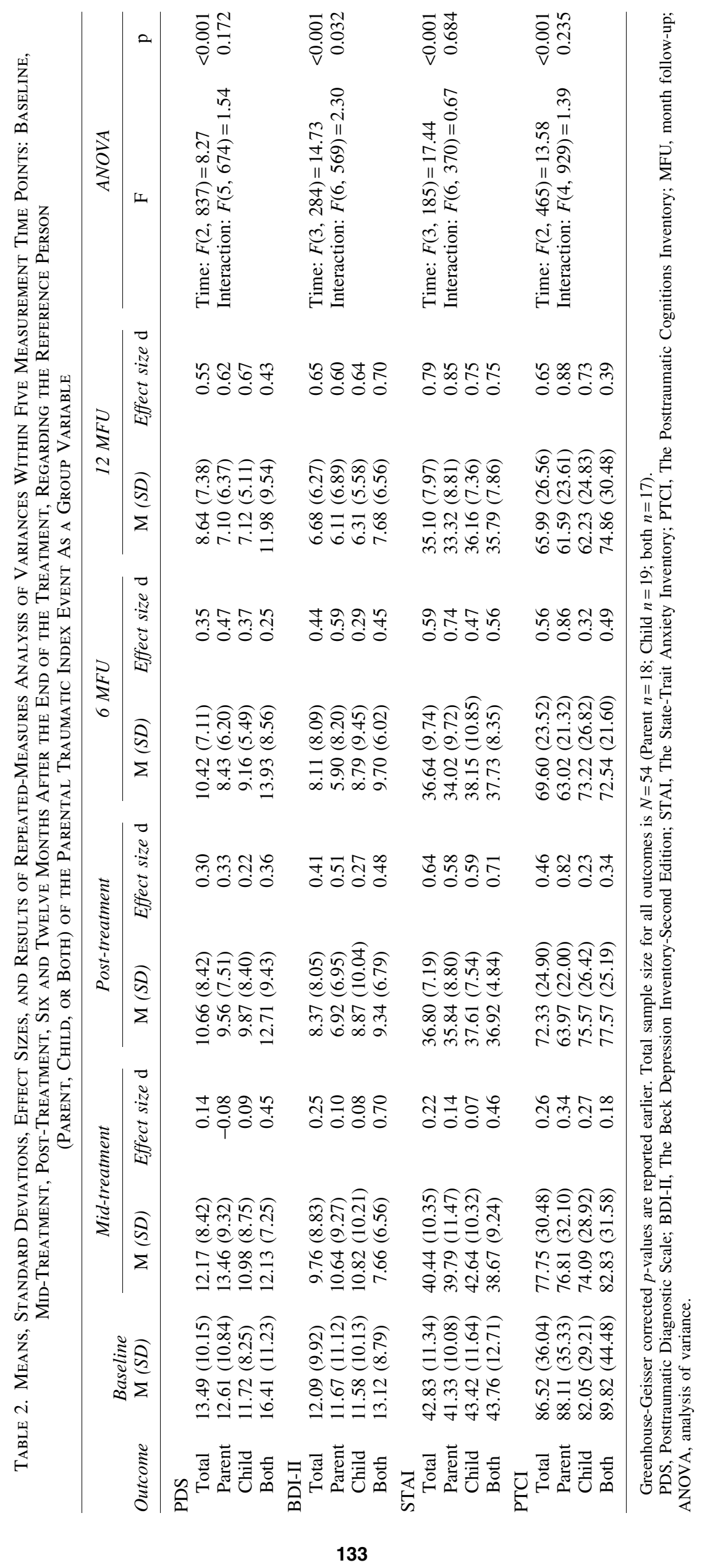


depression, anxiety, and dysfunctional PTCs. Our results suggest no impact of parental trauma history on parental benefit from participation in TF-CBT.

\section{Limitations and Future Research}

Several limitations of this study need to be addressed. Due to the lack of a control group, we cannot rule out the possibility that the effects at 6- and $12 \mathrm{MFU}$ might be attributable to spontaneous remission or alternative treatments. However, it has been reported elsewhere that parental symptoms stay stable for up to 30 months, if left untreated (Laor et al. 1997). Since we assessed parental variables only via questionnaires, we were not able to provide any information about parental diagnostic status. Because parents used the checklist to indicate those potentially traumatic events that they and their child had experienced or witnessed and based on the same check list reported on an index event, the "both" category might refer to the event, both of them experienced at the same time, or the similar event they experienced independently from each other. In the second case, it remains unclear whether parents rated their symptoms regarding both events, experienced independently from each other, or only one of those. In addition, if parents reported on more than one traumatic event, considering their own traumatic events and their child's lifetime trauma exposure, separating out the effects of different traumas retrospectively, while rating symptoms, might be fraught with potential measurement error. As our focus was on children, we did not ask parents whether they received any additional psychological treatment during the study participation. Another limitation was that our study had not included a measure of actual parenting behavior. A limited sample size, with a predominance of mothers in the study sample means that our findings should not be applied in a generalized manner to fathers of children with PTSS. Finally, our participants were parents of children at the age 6 years old and older, meaning that our results cannot be generalized to parents of preschool children.

Future studies should include a control group with an alternative treatment, assess parental diagnostic status, and include sufficient sample size with more fathers and parents of preschool children, to be able to determine an impact of parental trauma history on their response to TF-CBT. An impact of TF-CBT on participating parents, in the context of their trauma history, should not be investigated only for parental clinical variables. Other variables, such as parental emotional regulation, parenting efficacy, parent-child communication, and familial attachments should be taken into account in future studies. An interplay between parent and child' symptoms should be further investigated, especially in the context of the treatment response altogether and the long-term treatment effects and regarding children with complex PTSD, as proposed for International Classification of Diseases 11th Revision (Sachser et al. 2017). Further, a Norwegian study found that TF-CBT for children is likely to be a cost-effective alternative to treatment as usual (Aas et al. 2019). Therefore, future studies should investigate whether parental participation in TF-CBT reduces their own utilization of mental-health services and thereby reduces mental healthrelated costs. Finally, future studies should investigate parental benefit and their impact on a child's recovery in the context of another evidence-based trauma-focused treatment for children.

\section{Conclusion}

Although TF-CBT is a treatment developed for children, according to our results, it has also the potential to decrease symptoms and distress of parents involved in the child's therapy, with long- lasting effects. In line with other studies in the field of traumafocused care for children, our results suggest parental benefit from TF-CBT at post-treatment and sustainability of the treatment gains until 1-year post-treatment. Parental benefit from TF-CBT seems to be independent from the parental previous traumatic experiences. Therefore, it seems feasible to also include parents with their own trauma history or even the same type of trauma in TF-CBT.

\section{Clinical Significance}

The results of this study emphasize the advantage for parents from participating in TF-CBT delivered to their child. We know from other studies (e.g., Tutus and Goldbeck 2016) that parents of traumatized children frequently develop symptoms on their own, which might, in turn, affect a child's symptoms and treatment response. Therefore, clinicians treating children affected by trauma should be educated to regularly screen and monitor symptoms in their parents. Parents with persisting clinically relevant symptoms should then be referred to appropriate psychological and/or psychopharmacological treatment, if needed. In this case, a collaboration between clinicians treating parents and their children might essentially enhance the treatment effects in both. However, assuming an interplay between a parent's and a child's symptoms, more team work between clinicians treating both, children and their parents might be beneficial, also in the context of other mental health problems, and independently from traumatic experiences.

\section{Disclosures}

No competing financial interests exist.

\section{References}

Aas E, Iversen T, Holt T, Ormhaug SM, Jensen TK: Cost-effectiveness analysis of trauma-focused cognitive behavioral therapy: A randomized control trial among Norwegian youth. J Clin Child Adolesc Psychol 48(Suppl 1):298-311, 2019.

Alisic E, Zalta AK, Van Wesel F, Larsen SE, Hafstad GS, Hassanpour $\mathrm{K}$, Smid GE: Rates of post-traumatic stress disorder in traumaexposed children and adolescents: Meta-analysis. Br J Psychiatry 204:335-340, 2014.

American Psychiatric Association: Diagnostic and Statistical Manual of Mental Disorders, 4th ed. Washington, DC: American Psychiatric Association; 1994.

Beck AT, Steer RA, Brown GK: Beck Depression Inventory-Second Edition. Manual. San Antonio (Texas), The Psychological Corporation, 1996.

Cohen J: Statistical Power Analysis for the Behavioral Sciences. Hillsdale (New Jersey), Erlbaum, 1988.

Cohen J, Mannarino A, Knudsen K: Treating childhood traumatic grief: A pilot study. J Am Acad Child Adolesc Psychiatry 43:12251233, 2004a.

Cohen JA, Deblinger E, Mannarino AP, Steer RA: A multisite, randomized controlled trial for children with sexual abuse-related PTSD symptoms. J Am Acad Child Adolesc Psychiatry 43:393402, 2004b.

Cohen JA, Mannarino AP, Deblinger E: Treating Trauma and Traumatic Grief in Children and Adolescents. New York, Guilford, 2006a.

Cohen JA, Mannarino AP, Deblinger E: Traumafokussierte kognitive Verhaltenstherapie bei Kindern und Jugendlichen. Heidelberg, Germany, Springer Medizin Verlag, 2009.

Cohen JA, Mannarino AP, Knudsen K: Treating sexually abused children: 1 year follow-up of a randomized controlled trial. Child Abuse Neglect 29:135-145, 2005. 
Cohen JA, Mannarino AP, Perel JM, Staron V: A pilot randomized controlled trial of combined trauma-focused CBT and sertraline for childhood PTSD symptoms. J Am Acad Child Adolesc Psychiatry 46:811-819, 2007.

Cohen JA, Mannarino AP, Staron VR: A pilot study of modified cognitive-behavioral therapy for childhood traumatic grief (CBTCTG). J Am Acad Child Adolesc Psychiatry 45:1465-1473, 2006b.

Deblinger E, Lippmann J, Steer RA: Sexually abused children suffering posttraumatic stress symptoms: Initial treatment outcome findings. Child Maltreat 1:310-321, 1996.

Deblinger E, Mannarino AP, Cohen JA, Runyon MK, Steer RA: Trauma-focused cognitive behavioral therapy for children: Impact of the trauma narrative and treatment length. Depress Anxiety 28: 67-75, 2011.

Deblinger E, Mannarino AP, Cohen JA, Steer RA: A follow-up study of a multisite, randomized, controlled trial for children with sexual abuse-related PTSD symptoms. J Am Acad Child Adolesc Psychiatry 45:1474-1484, 2006.

Deblinger E, Stauffer L, Steer RA: Comparative efficacies of supportive and cognitive behavioral group therapies for young children who have been sexually abused and their nonoffending mothers. Child Maltreat 6:332-343, 2001.

Deblinger E, Steer RA, Lippmann J: Two-year follow-up study of cognitive behavioral therapy for sexually abused children suffering post-traumatic stress symptoms. Child Abuse Negelct 23:13711378, 1999.

Dempster AP, Laird NM, Rubin DB: Maximum likelihood from incomplete data via the EM algorithm. J R Stat Soc Ser B (Methodol) 39:1-38, 1977.

Dorsey S, Briggs EC, Woods BA: Cognitive-behavioral treatment for posttraumatic stress disorder in children and adolescents. Child Adolesc Psychiatr Clin N Am 20:255-269, 2011.

Dorsey S, McLaughlin KA, Kerns SE, Harrison JP, Lambert HK, Briggs EC, Cox JR, Amaya-Jackson L: Evidence base update for psychosocial treatments for children and adolescents exposed to traumatic events. J Clin Child Adolesc Psychol 46:303-330, 2017.

Foa EB: Posttraumatic Stress Diagnostic Scale (PDS). Manual. Minneapolis, MI: National Computer Systems, Inc., 1995.

Foa EB, Ehlers A, Clark DM, Tolin DF, Orsillo SM: The PostTraumatic Cognitions Inventory (PTCI): Development and validation. Psychol Assess 11:303-314, 1999.

Goldbeck L, Muche R, Sachser C, Tutus D, Rosner R: Effectiveness of Trauma-focused Cognitive Behavioural Therapy (Tf-CBT) for children and adolescents: A randomized controlled trial in eight German mental health clinics. Psychother Psychosom 85:159-170, 2016.

Hiller RM, Meiser-Stedman R, Lobo S, Creswell C, Fearon P, Ehlers A, Murray L, Halligan SL: A longitudinal investigation of the role of parental responses in predicting children's post-traumatic distress. J Child Psychol Psychiatry 59:781-789, 2018.

Holt T, Jensen TK, Wentzel-Larsen T: The change and the mediating role of parental emotional reactions and depression in the treatment of traumatized youth: Results from a randomized controlled study. Child Adolesc Psychiatry Ment Health 8:11, 2014.

International Society for Traumatic Stress Studies (ISTSS): ISTSS PTSD Guidelines-Methodology and Recommendations. 2019. Available at: https://istss.org/clinical-resources/treating-trauma/ new-istss-prevention-and-treatment-guidelines (accessed December 9, 2019).

Jensen TK, Holt T, Ormhaug SM: A follow-up study from a multisite, randomized controlled trial for traumatized children receiving TFCBT. J Abnorm Child Psychol 45:1587-1597, 2017.

Jensen TK, Holt T, Ormhaug SM, Fjermestad KW, Wentzel-Larsen T: Change in post-traumatic cognitions mediates treatment effects for traumatized youth-a randomized controlled trial. J Couns Psychol 65:166-177, 2018

King NJ, Tonge BJ, Mullen P, Myerson N, Heyne D, Rollings S, Martin R, Ollendick TH: Treating sexually abused children with posttraumatic stress symptoms: A randomized clinical trial. J Am Acad Child Adolesc Psychiatry 39:1347-1355, 2000.

Koplewicz HS, Vogel JM, Solanto MV, Morrissey RF, Alonso CM, Abikoff H, Gallagher R, Novick RM: Child and parent response to the 1993 World Trade Center bombing. J Trauma Stress 15:77-85, 2002.

Kühner C, Bürger C, Keller F, Hautzinger M: Reliability and validity of the revised Beck Depression Inventory (BDI-II). Findings from German-speaking samples [in German]. Nervenarzt 78:651-656, 2007.

Laor N, Wolmer L, Mayes LC, Gershon A, Weizman R, Cohen DJ: Israeli preschool children under scuds: A 30-month follow-up. J Am Acad Child Adolesc Psychiatry 36:349-356, 1997.

Laux L, Glanzmann P, Schaffner P, Spielberger CD: Das State-Trait Angstinventar. Göttingen, Germany, Beltz Test GmbH, 1981.

Mannarino AP, Cohen JA, Deblinger E: Trauma-focused cognitivebehavioral therapy for children: Sustained impact of treatment 6 and 12 months later. Child Maltreat 17:231-241, 2012.

Martin CG, Everett Y, Skowron EA, Zalewski M: The role of caregiver psychopathology in the treatment of childhood trauma with trauma-focused cognitive behavioral therapy: A systematic review. Clin Child Fam Psychol Rev 22:273-289, 2019.

Meiser-Stedman R, Smith P, Bryant R, Salmon K, Yule W, Dalgleish T, Nixon RD: Development and validation of the Child PostTraumatic Cognitions Inventory (CPTCI). J Child Psychol Psychiatry 50:432-440, 2009.

Morina N, Koerssen R, Pollet TV: Interventions for children and adolescents with posttraumatic stress disorder: A meta-analysis of comparative outcome studies. Clin Psychol Rev 47:41-54, 2016.

Morris A, Gabert-Quillen C, Delahanty D: The association between parent PTSD/depression symptoms and child PTSD symptoms: A meta-analysis. J Pediatr Psychol 37:1076-1088, 2012.

Nader K, Kriegler J, Blake D: The Clinican-Administered PTSD Scale for Children and Adolescents for DSM-IV (CAPS-CA). White River Junction (Vermont), National Centre for PTSD, 2002.

Neill EL, Weems CF, Scheeringa MS: CBT for child PTSD is associated with reductions in maternal depression: Evidence for bidirectional effects. J Clin Child Adolesc Psychol 47:410-420, 2018. Nixon RD, Sterk J, Pearce A: A randomized trial of cognitive behaviour therapy and cognitive therapy for children with posttraumatic stress disorder following single-incident trauma. J Abnorm Child Psychol 40:327-337, 2012.

Nixon RD, Sterk J, Pearce A, Weber N: A randomized trial of cognitive behavior therapy and cognitive therapy for children with posttraumatic stress disorder following single-incident trauma: Predictors and outcome at 1-year follow-up. Psychol Trauma 9: 471-478, 2017.

Pfeiffer E, Sachser C, de Haan A, Tutus D, Goldbeck L: Dysfunctional posttraumatic cognitions as a mediator of symptom reduction in trauma-focused cognitive behavioral therapy with children and adolescents: Results of a randomized controlled trial. Behav Res Ther 97:178-182, 2017.

Sachser C, Keller F, Goldbeck L: Complex PTSD as proposed for ICD-11: Validation of a new disorder in children and adolescents and their response to trauma-focused cognitive behavioral therapy. J Child Psycol Psychiatry 58:160-168, 2017.

Scheeringa MS, Myers L, Putnam FW, Zeanah CH: Maternal factors as moderators or mediators of PTSD symptoms in very young children: A two-year prospective study. J Fam Violence 30:633642, 2015. 
Scheeringa MS, Zeanah CH: A relational perspective on PTSD in early childhood. J Trauma Stress 14:799-815, 2001.

Scher CD, Suvak MK, Resick PA: Trauma cognitions are related to symptoms up to 10 years after cognitive behavioral treatment for posttraumatic stress disorder. Psychol Trauma 9:750-757, 2017.

Silverman WK, Hinshaw SP: The second special issue on evidencebased psychosocial treatments for children and adolescents: A 10year update. J Clin Child Adolesc Psychol 37:1-7, 2008.

Smoller JW: The genetics of stress-related disorders: PTSD, depression, and anxiety disorders. Neuropsychopharmacology 41:297319, 2016.

Spielberger CD, Gorsuch RL, Lushene RE: Manual for the Stait-Trait Anxiety Inventory. Santa Clara, CA: Palo Alto, Consulting Psychologists Press, 1970.

Trickey D, Siddaway AP, Meiser-Stedman R, Serpell L, Field AP: A meta-analysis of risk factors for post-traumatic stress disorder in children and adolescents. Clin Psychol Rev 32:122-138, 2012.

Tutus D, Goldbeck L: Posttraumatic symptoms and cognitions in parents of children and adolescents with PTSD. Eur Child Adolesc Psychiatry 25:997-1005, 2016.

Tutus D, Goldbeck L, Pfeiffer E, Sachser C, Plener PL: Parental dysfunctional posttraumatic cognitions in trauma-focused cognitive behavioral therapy for children and adolescents. Psychol Trauma 11:722-731, 2018.
Tutus D, Keller F, Sachser C, Pfeiffer E, Goldbeck L: Change in parental depressive symptoms in trauma-focused cognitive-behavioral therapy: Results from a randomized controlled trial. J Child Adolesc Psychopharmacol 27:200-205, 2017a.

Tutus D, Pfeiffer E, Sachser C, Rosner R, Goldbeck L: Sustainability of treatment effects of trauma-focused cognitive-behavioral therapy (TF-CBT) for children and adolescents: Findings from 6- and 12month follow-ups. Psychother Psychosom 86:379-381, $2017 \mathrm{~b}$.

Zalta AK, Gillihan SJ, Fisher AJ, Mintz J, McLean CP, Yehuda R, Foa EB: Change in negative cognitions associated with PTSD predicts symptom reduction in prolonged exposure. J Consult Clin Psychol 82:171-175, 2014.

Address correspondence to:

Dunja Tutus, MSc

Department of Child and Adolescent Psychiatry/Psychotherapy

Ulm University

Steinhoevelstr. 1

Ulm 89075

Germany

E-mail: dunja.tutus@uniklinik-ulm.de 